\title{
Evaluation of cumulative prognostic score based on pretreatment plasma fibrinogen and serum albumin levels in patients with newly diagnosed high-grade gliomas
}

\author{
Zhen-Qiang He ${ }^{1, *}$, Hao Duan ${ }^{1, *}$, Chao $\mathrm{Ke}^{1}$, Xiang-Heng Zhang ${ }^{1}$, Cheng-Cheng Guo ${ }^{1}$, \\ Fuad Al-Nahari ${ }^{1}$, Ji Zhang ${ }^{1}$, Zheng-He Chen ${ }^{1}$, Yin-Sheng Chen ${ }^{1}$, Zhi-Gang Liu ${ }^{2}$, Jian \\ Wang $^{1}$, Zhong-Ping Chen ${ }^{1}$, Xiao-Bing Jiang ${ }^{1}$ and Yong-Gao Mou ${ }^{1}$ \\ ${ }^{1}$ Department of Neurosurgery/Neuro-Oncology, Sun Yat-Sen University Cancer Center, State Key Laboratory of Oncology in \\ South China, Collaborative Innovation Center for Cancer Medicine, Guangzhou, 510060, China \\ ${ }^{2}$ Key Laboratory of Translational Radiation Oncology, Hunan Province, Department of Radiation Oncology, Hunan Cancer \\ Hospital, The Affiliated Cancer Hospital of Xiangya School of Medicine, Central South University, Changsha, 410013, China \\ *These authors contributed equally to this work \\ Correspondence to: Yong-Gao Mou, email: mouyg@sysucc.org.cn \\ Xiao-Bing Jiang, email: jiangxiaob1@sysucc.org.cn
}

Keywords: high-grade gliomas, fibrinogen, albumin, cumulative score, prognosis

Received: March 10, 2017 Accepted: April 29, $2017 \quad$ Published: May 13, 2017

Copyright: He et al. This is an open-access article distributed under the terms of the Creative Commons Attribution License 3.0 (CC BY 3.0), which permits unrestricted use, distribution, and reproduction in any medium, provided the original author and source are credited.

\section{ABSTRACT}

This retrospective study was designed to determine the prognostic value of a cumulative score (FA score) based on pretreatment plasma fibrinogen and serum albumin levels for 326 patients newly diagnosed high-grade glioma (HGG). Receiver operating characteristic (ROC) curve analysis was performed to determine the optimal cut-off values. Univariate and multivariate analysis were performed to evaluate the independent prognostic value of the FA scores associated with overall survival (OS) and progression-free survival (PFS). The optimal cut-off values were $2.815 \mathrm{~g} / \mathrm{L}$ for fibrinogen and $43.65 \mathrm{~g} / \mathrm{L}$ for albumin. PFS and OS were significantly worse for patients with higher FA scores. Patients with elevated fibrinogen level and decreased albumin levels had 3.00-fold higher risk of tumor progression and had a 3.23-fold higher risk of death compared with those with normal values. Multivariate analysis demonstrated FA score was an independent predictive factor for PFS and OS. Moreover, PFS and OS were better for the patients with lower FA score, either in patients with grade III or IV gliomas. These findings indicated that the pretreatment FA score could serve as a simple and noninvasive marker to predict the prognosis of patients with HGG.

\section{INTRODUCTION}

High-grade gliomas (HGG), defined as World Health Organization (WHO) Grade III and IV gliomas, are the most devastating malignancies in the central nerve system. HGG accounts for $>60 \%$ of all gliomas and is characterized by high morbidity and mortality because of tumor localization and locally invasive nature $[1,2]$. Despite aggressive treatment modalities, including tumor resection accompanied by fractionated radiotherapy and temozolomide-based chemotherapy, the median survival for patients with glioblastoma and anaplastic gliomas are $12-14$ months and $2-5$ years, respectively $[2,3]$. To ensure optimal adjuvant treatment and intense follow-up for patients with $\mathrm{HGG}$, a simple and instructive marker is required to predict postoperative survival.

The histopathological characteristics, performance status, tumor grade, prognostic indicators based on systemic inflammation levels and nutritional conditions of patients with HGG are published [4, 5]. Fibrinogen, a 340-kD liver glycoprotein, has been reported as a key regulator in both inflammation and cancer progression [6]. Moreover, fibrinogen plays an important role in tumor cell proliferation, migration and angiogenesis [6]. Further, high pretreatment plasma fibrinogen levels are significantly associated with shorter survival in rectal cancer, non-small cell lung cancer, renal cell carcinoma and breast cancers [7-10]. Serum albumin levels are an important indicator 
of host's systemic inflammatory response and nutritional status as a prognostic indicator in several cancer [11-13]. Moreover, low serum albumin levels are associated with significantly short survival in glioblastoma patients [14].

The fibrinogen and albumin score (FA score), a novel cumulative prognostic score based on pretreatment fibrinogen and albumin levels, was firstly proposed by Matsuda et al. as a new prognostic scoring system in esophageal cancer patients treated with transthoracic esophagectomy [15]. The FA score is of outstanding prognostic value in esophageal cancer patients compared with Glasgow Prognostic Score (GPS) [15]. However, whether FA score predicts the survive of patients with HGG is unknown. We therefore performed a retrospective analysis to evaluate the prognostic performance of the FA score in a relatively large cohort of patients with HGG.

\section{RESULTS}

\section{Patient characteristics}

The characteristics of $326 \mathrm{HGG}$ patients are shown in Table 1. The median follow-up was 20.0 months (interquartile range [IQR]: 13.2-36.2 months), mean age was 44.0 years (range: 5-78 years), and 60.4\% (197/326) of patients were males. Gross total resection (GTR) and subtotal resection (STR) was achieved for $63.5 \%$ and $27.3 \%$ of patients, respectively, and $8.6 \%$ underwent partial resection and $0.6 \%$ patients received biopsy only. Aggressive adjuvant treatment of 179 patients $(54.9 \%)$ included fractionated radiotherapy and first-line chemotherapy. The treatment modalities administered to patients were summarized in Table 2 .

\section{Cut-off determination of fibrinogen and albumin}

The mean pretreatment plasma fibrinogen and serum albumin levels were $2.97 \pm 1.06 \mathrm{~g} / \mathrm{L}$ (range: $1.39-13.00$ $\mathrm{g} / \mathrm{L}$ ) and $43.37 \pm 3.80 \mathrm{~g} / \mathrm{L}$ (range: $32.90-64.39 \mathrm{~g} / \mathrm{L}$ ). The areas under the receiver operating characteristic (ROC) curve were 0.611 for fibrinogen and 0.605 for albumin. Using ROC analysis, the optimal cut-off values for fibrinogen was $2.815 \mathrm{~g} / \mathrm{L}$ and $43.65 \mathrm{~g} / \mathrm{L}$ for albumin, respectively. Among the 326 patients, 151 (46.3\%) patients had elevated pretreatment fibrinogen levels $\geq 2.815 \mathrm{~g} / \mathrm{L}$, and $172(52.8 \%)$ patients had decreased pretreatment albumin levels $\leq 43.65 \mathrm{~g} / \mathrm{L}$. Therefore, 79 (24.2\%), 171 $(52.5 \%)$, and $76(23.3 \%)$ patients had FA scores $=0,1$ and 2 , respectively (Table 1 ).

\section{Association between clinicopathological features and FA score}

The clinicopathological features of all patients stratified according to FA scores are shown in Table 1. Higher pretreatment FA score were observed for patient of advanced age $(p<0.001)$ and a higher glioma grade $(p<0.007)$. However, there was no significant association between FA score and sex, Karnofsky performance status (KPS), tumor size, tumor location, extent of resection, or treatment modality.

\section{Survival analysis}

At the date of final follow-up, 254 (77.9\%) patients had died. The median OS of all the patients was 17.23 months (95\% CI $=15.14-19.33$ months), and 25.53 months $(95 \% \mathrm{CI}=17.03-34.04$ months $)$ and 14.30 months $(95 \% \mathrm{CI}=12.22-16.38$ months) for those with WHO Grades III and IV gliomas, respectively. KaplanMeier analysis revealed that that the pretreatment FA score was negatively associated with PFS $(p<0.001)$ and OS $(p<0.001)$ (Figure 1). When we conducted subgroup analysis of prognosis after stratifying patients according to tumor grade, we found that the pretreatment FA score retained its predictive value for PFS and OS for patients with grades III (PFS, $p<0.001$; OS, $p<$ 0.001 ) and IV (PFS, $p=0.002$; OS, $p=0.001$ ) disease, respectively (Figure 2A-2D). We next performed Kaplan-Meier analysis of patients stratified according to treatment modalities. As shown in Figure 2E-2H, a high pretreatment FA score significantly predicted worse PFS and OS of patients who did (PFS, $p=0.009$; OS, $p=0.004$ ) or did not (PFS, $p<0.001$; OS, $p<0.001$ ) receive aggressive treatment.

\section{Univariate and multivariate Cox regression analysis}

Univariate analysis revealed that pretreatment FA score, as well as traditionally predictors including age, KPS, tumor grade, extent of resection and aggressive treatment were significantly associated with both OS and PFS (Table 3), and patients with FA score $=2$ had 3.00-fold higher risk of tumor progression and a 3.23-fold higher risk of death compared with those with FA score $=0$. Furthermore, multivariate Cox regression analysis revealed that higher pretreatment FA score still predicted worse PFS $(p<0.001)$ and OS $(p<0.001)$ independent of age, tumor grade, extent of resection or aggressive treatment (Table 4).

\section{DISCUSSION}

In the present study, we retrospectively analyzed 326 consecutive patients with high-grade gliomas who were treated with surgery and adjuvant therapies. Using the cut-off values of pretreatment fibrinogen and albumin determined from ROC curves, we examined the prognostic value of a cumulative prognostic score (pretreatment FA score). The results are as follows: (1) A higher pretreatment FA score was significantly associated 
Table 1: The clinicopathological features stratified by pretreatment FA score $(n=326)$

\begin{tabular}{|c|c|c|c|c|c|}
\hline Variables & All patients & $\begin{array}{l}\text { FA score } 0 \\
(n=79)\end{array}$ & $\begin{array}{l}\text { FA score } 1 \\
(n=171)\end{array}$ & $\begin{array}{c}\text { FA score } 2 \\
(n=76)\end{array}$ & $P$ value \\
\hline Age [years; $n(\%)]$ & & & & & $<0.001$ \\
\hline$<60$ & $269(82.5)$ & 75 (27.9) & $140(52.0)$ & $54(20.1)$ & \\
\hline$\geq 60$ & $57(17.5)$ & $4(7.0)$ & $31(54.4)$ & $22(38.6)$ & \\
\hline Gender $[n(\%)]$ & & & & & 0.286 \\
\hline Male & $198(60.7)$ & $37(28.9)$ & $63(49.2)$ & $28(21.9)$ & \\
\hline Female & $128(39.3)$ & $42(21.2)$ & $108(54.5)$ & $48(24.2)$ & \\
\hline $\operatorname{KPS}[n(\%)]$ & & & & & 0.727 \\
\hline$\geq 70$ & $303(92.9)$ & $75(24.8)$ & $158(52.1)$ & $70(23.1)$ & \\
\hline$<70$ & $23(7.1)$ & $4(17.4)$ & $13(56.5)$ & $6(26.1)$ & \\
\hline Tumor grade $[n(\%)]$ & & & & & 0.007 \\
\hline WHO III & $157(48.2)$ & $48(30.6)$ & $82(52.2)$ & $27(17.2)$ & \\
\hline WHO IV & $169(51.8)$ & $31(18.3)$ & $89(52.7)$ & $49(29.0)$ & \\
\hline Tumor size $[\mathrm{cm} ; n(\%)]$ & & & & & 0.220 \\
\hline$\leq 5$ & $156(47.9)$ & $42(26.9)$ & $74(47.4)$ & $40(25.6)$ & \\
\hline$>5$ & $170(52.1)$ & $37(21.8)$ & $97(57.1)$ & $36(21.2)$ & \\
\hline Tumor location $[n(\%)]$ & & & & & 0.079 \\
\hline Cerebral cortex & $291(89.3)$ & 66 (22.7) & $153(52.6)$ & $72(24.7)$ & \\
\hline Non cerebral cortex & $35(10.7)$ & $13(37.1)$ & $18(51.4)$ & $4(11.4)$ & \\
\hline Extent of resection $[n(\%)]$ & & & & & 0.829 \\
\hline Gross total resection & $207(63.5)$ & $52(25.1)$ & $108(52.2)$ & $47(22.7)$ & \\
\hline Subtotal resection & $89(27.3)$ & $18(20.2)$ & $49(55.1)$ & $22(24.7)$ & \\
\hline Partial resection and biopsy & $30(9.2)$ & $9(30.0)$ & $14(46.7)$ & $7(23.3)$ & \\
\hline Aggressive treatment $[n(\%)]$ & & & & & 0.122 \\
\hline Yes & $183(56.1)$ & $48(26.2)$ & $100(54.6)$ & $35(19.1)$ & \\
\hline No & $143(43.9)$ & $31(21.7)$ & $71(49.7)$ & $41(28.7)$ & \\
\hline $\begin{array}{l}\text { Pretreatment plasma fibrinogen level } \\
{[\mathrm{g} / \mathrm{L} ; \text { mean } \pm \mathrm{SD}]}\end{array}$ & $2.97 \pm 1.06$ & $2.34 \pm 0.30$ & $2.90 \pm 1.13$ & $3.78 \pm 0.87$ & $<0.001$ \\
\hline $\begin{array}{l}\text { Pretreatment serum albumin level } \\
{[\mathrm{g} / \mathrm{L} ; \text { mean } \pm \mathrm{SD}]}\end{array}$ & $43.37 \pm 3.80$ & $46.74 \pm 2.85$ & $43.09 \pm 3.54$ & $40.52 \pm 2.28$ & $<0.001$ \\
\hline
\end{tabular}

FA score, fibrinogen and albumin score; n, number; KPS, Karnofsky performance status; WHO, World Health Organization;

with advanced age and higher tumor grade. (2) The pretreatment FA score was an independent prognostic factor of PFS and OS of patients with high-grade gliomas. (3) The prognostic value of the FA score was significant in subgroups that included patients diagnosed with WHO Grade III or IV as well as patients who did or did not undergo aggressive treatment.

This novel cumulative prognostic score proposed by Matsuda et al is based on pretreatment plasma fibrinogen and serum albumin levels [15] which serve as prognostic markers of solid cancers $[6,13]$. The adverse effect of elevated pretreatment plasma fibrinogen on survival is experienced in patients with colon cancer[11], lung cancer [8], breast cancer [10], renal cell carcinoma[16] and ovarian cancer [17]. However, the prognostic value of pretreatment fibrinogen levels in patients with high-grade glioma was previously unknown. In contrast, pretreatment serum albumin levels are prognostic for patients with glioblastoma. Schwartzbaum et al and Borg et al reported that a low preoperative serum albumin level is a significant predictor of poor overall survival in patients with glioblastoma multiforme (GBM) [14, 18].

Here we show that the FA score showed significant prognostic value in HGG patients. Patients with a FA score $=1$ had 2.406-fold higher risk of death than those with a FA score of 0 . And patients with a FA score of 2 had a 3.032-fold higher risk of death than those with a FA score $=0$ in the multivariate analysis. Therefore, $\mathrm{HGG}$ patients with a higher FA score before surgery may require aggressive treatment and intense follow-up. 
Table 2: Treatment modality for the patients $(n=326)$

\begin{tabular}{ll}
\hline Treatment modality & \multicolumn{1}{c}{$\boldsymbol{N ( \% )}$} \\
\hline Surgical resection only & $104(31.9)$ \\
Surgery $\rightarrow$ Radiotherapy & $32(9.8)$ \\
Surgery $\rightarrow$ Radiotherapy $\rightarrow$ Chemotherapy (A) & $129(39.6)$ \\
Surgery $\rightarrow$ Radiotherapy $\rightarrow$ Chemotherapy (B) & $50(15.3)$ \\
Surgery $\rightarrow$ Chemotherapy (A) & $6(1.8)$ \\
Surgery $\rightarrow$ Chemotherapy (B) & $5(1.5)$ \\
\hline
\end{tabular}

A, Temozolomide-based; B, nitrosourea-based or platinum-based.

Table 3: The univariate analysis of the prognostic factors for PFS and OS $(n=326)$

\begin{tabular}{|c|c|c|c|c|c|c|}
\hline & \multicolumn{3}{|c|}{ Progression-free survival } & \multicolumn{3}{|c|}{ Overall survival } \\
\hline & HR & $95 \%$ CI & $P$ value & HR & $95 \%$ CI & $P$ value \\
\hline Age, years & & & $<0.001$ & & & $<0.001$ \\
\hline$<60$ & 1 & Referent & & 1 & Referent & \\
\hline$\geq 60$ & 2.184 & $1.614-2.954$ & & 2.095 & $1.537-2.854$ & \\
\hline Gender & & & 0.039 & & & 0.140 \\
\hline Male & 1 & Referent & & 1 & Referent & \\
\hline Female & 0.770 & $0.601-0.987$ & & 0.826 & $0.640-1.065$ & \\
\hline KPS & & & $<0.001$ & & & 0.002 \\
\hline$\geq 70$ & 1 & Referent & & 1 & Referent & \\
\hline$<70$ & 2.014 & $1.308-3.103$ & & 1.966 & $1.277-3.026$ & \\
\hline Tumor grade & & & $<0.001$ & & & $<0.001$ \\
\hline WHO grade III & 1 & Referent & & 1 & Referent & \\
\hline WHO grade IV & 1.676 & $1.313-2.139$ & & 1.772 & $1.375-2.283$ & \\
\hline Tumor size & & & 0.277 & & & 0.201 \\
\hline$\leq 5 \mathrm{~cm}$ & 1 & Referent & & 1 & Referent & \\
\hline$>5 \mathrm{~cm}$ & 0.876 & $0.690-1.112$ & & 0.851 & $0.666-1.089$ & \\
\hline Tumor location & & & 0.065 & & & 0.072 \\
\hline Cerebral cortex & 1 & Referent & & 1 & Referent & \\
\hline Non cerebral cortex & 1.409 & $0.987-2.030$ & & 1.413 & $0.969-2.058$ & \\
\hline Extent of resection & & & $<0.001$ & & & $<0.001$ \\
\hline Gross total resection & 1 & Referent & & 1 & Referent & \\
\hline Subtotal resection & 1.656 & $1.268-2.163$ & 0.001 & 1.587 & $1.206-2.090$ & 0.001 \\
\hline $\begin{array}{l}\text { Partial resection and } \\
\text { biopsy }\end{array}$ & 3.734 & $2.486-5.607$ & $<0.001$ & 3.536 & $2.330-5.365$ & $<0.001$ \\
\hline Aggressive treatment & & & $<0.001$ & & & $<0.001$ \\
\hline Yes & 1 & Referent & & 1 & Referent & \\
\hline No & 1.889 & $1.486-2.403$ & & 2.224 & $1.736-2.850$ & \\
\hline FA score & & & $<0.001$ & & & $<0.001$ \\
\hline 0 & 1 & Referent & & 1 & Referent & \\
\hline 1 & 2.017 & $1.464-2.777$ & $<0.001$ & 1.988 & $1.424-2.776$ & $<0.001$ \\
\hline 2 & 2.996 & $2.064-4.350$ & $<0.001$ & 3.233 & $2.198-4.754$ & $<0.001$ \\
\hline
\end{tabular}

HR, hazard ratio; CI, confidence interval; KPS, Karnofsky performance status; WHO, World Health Organization; FA score, fibrinogen and albumin score 
Table 4: The multivariate analysis of the prognostic factors for PFS and OS $(n=326)$

\begin{tabular}{|c|c|c|c|c|c|c|}
\hline & \multicolumn{3}{|c|}{ Progression-free survival } & \multicolumn{3}{|c|}{ Overall survival } \\
\hline & HR & $95 \% \mathrm{CI}$ & $P$ value & HR & $95 \% \mathrm{CI}$ & $P$ value \\
\hline Age, years & & & 0.003 & & & 0.015 \\
\hline$<60$ & 1 & Referent & & 1 & Referent & \\
\hline$\geq 60$ & 1.614 & $1.175-2.218$ & & 1.496 & $1.080-2.073$ & \\
\hline KPS & & & 0.102 & & & 0.152 \\
\hline$\geq 70$ & 1 & Referent & & 1 & Referent & \\
\hline$<70$ & 1.460 & $0.928-2.301$ & & 1.393 & $0.885-2.194$ & \\
\hline Tumor grade & & & $<0.001$ & & $<0.001$ & $<0.001$ \\
\hline WHO grade III & 1 & Referent & & 1 & Referent & \\
\hline WHO grade IV & 1.593 & $1.234-2.055$ & & 1.715 & $1.318-2.232$ & \\
\hline Extent of resection & & & $<0.001$ & & & $<0.001$ \\
\hline Gross total resection & 1 & Referent & & 1 & Referent & \\
\hline Subtotal resection & 1.613 & $1.222-2.129$ & 0.001 & 1.562 & $1.171-2.084$ & 0.002 \\
\hline $\begin{array}{l}\text { Partial resection and } \\
\text { biopsy }\end{array}$ & 5.324 & $3.467-8.175$ & $<0.001$ & 5.165 & $3.331-8.008$ & $<0.001$ \\
\hline Aggressive treatment & & & $<0.001$ & & & $<0.001$ \\
\hline Yes & 1 & Referent & & 1 & Referent & \\
\hline No & 2.059 & $1.608-2.637$ & & 2.457 & $1.905-3.169$ & \\
\hline FA score & & & $<0.001$ & & & $<0.001$ \\
\hline 0 & 1 & Referent & & 1 & Referent & \\
\hline 1 & 2.041 & $1.464-2.846$ & $<0.001$ & 2.046 & $1.447-2.894$ & $<0.001$ \\
\hline 2 & 2.792 & $1.890-4.123$ & $<0.001$ & 3.032 & $2.026-4.536$ & $<0.001$ \\
\hline
\end{tabular}

HR, hazard ratio; CI, confidence interval; KPS, Karnofsky performance status; WHO, World Health Organization; FA score, fibrinogen and albumin score
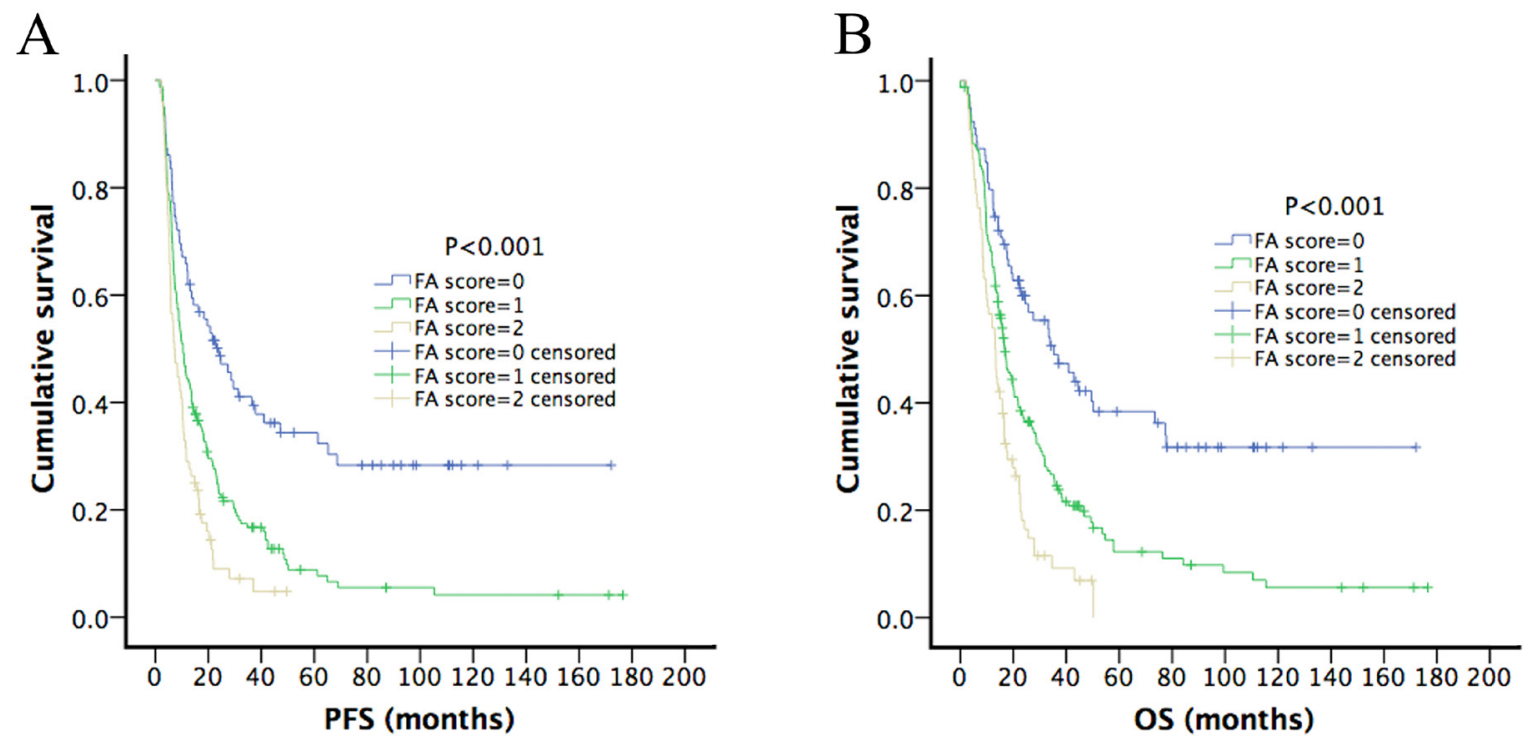

Figure 1: Kaplan-Meier survival curves of HGG patients. According to the optimal cut-off value of pretreatment plasma fibrinogen and serum albumin levels, we build up a cumulative score (FA score) and HGG patients were divided into three groups. Patients with elevated fibrinogen level $(\geq 2.815 \mathrm{~g} / \mathrm{L})$ and decreased albumin level $(\leq 43.65 \mathrm{~g} / \mathrm{L})$ were allocated to a score of 2 , those with only one of these abnormalities were allocated to a score of 1 and those with neither of these abnormalities were allocated a score of 0 . Pretreatment FA score is significantly predictive of DFS and OS, with higher FA scores among patients with shorter PFS $(\mathbf{A}, P<0.001)$ and OS $(\mathbf{B}, P<0.001)$. 

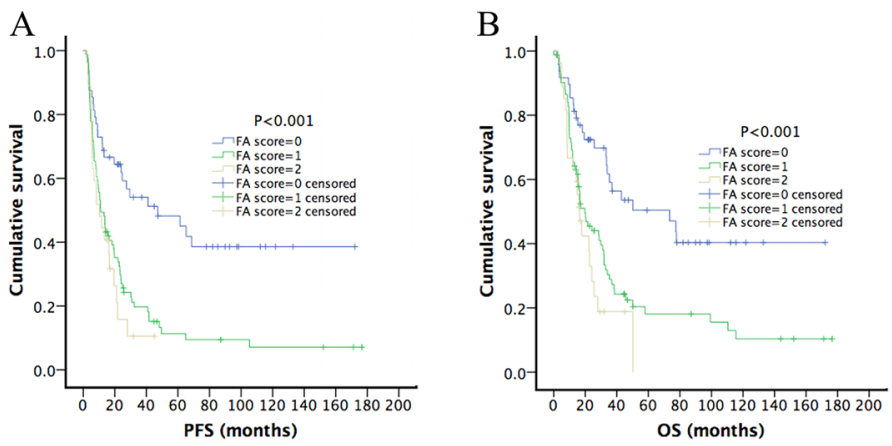

WHO Grade III gliomas
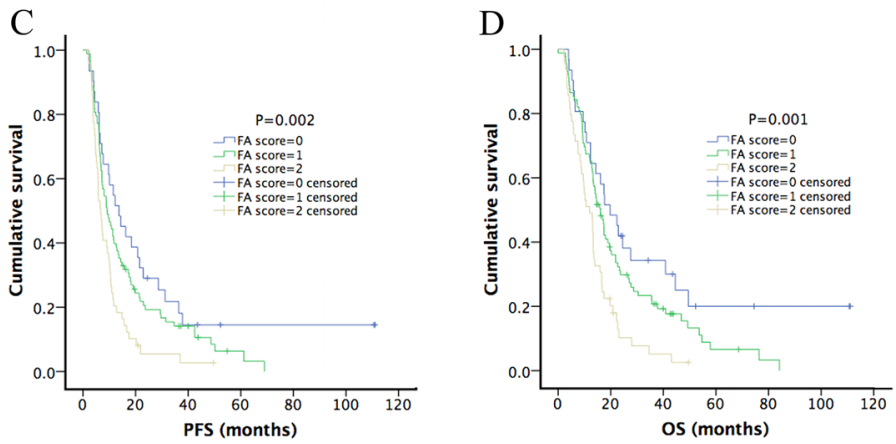

WHO Grade IV gliomas
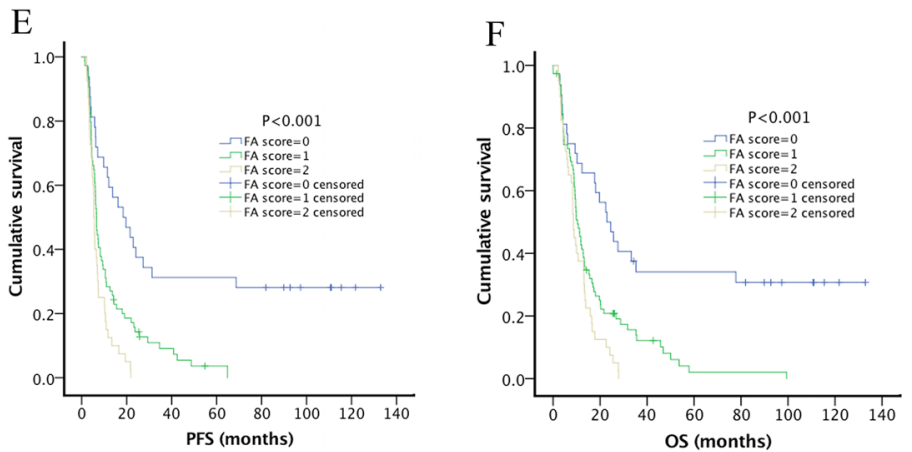

Without aggressive treatment
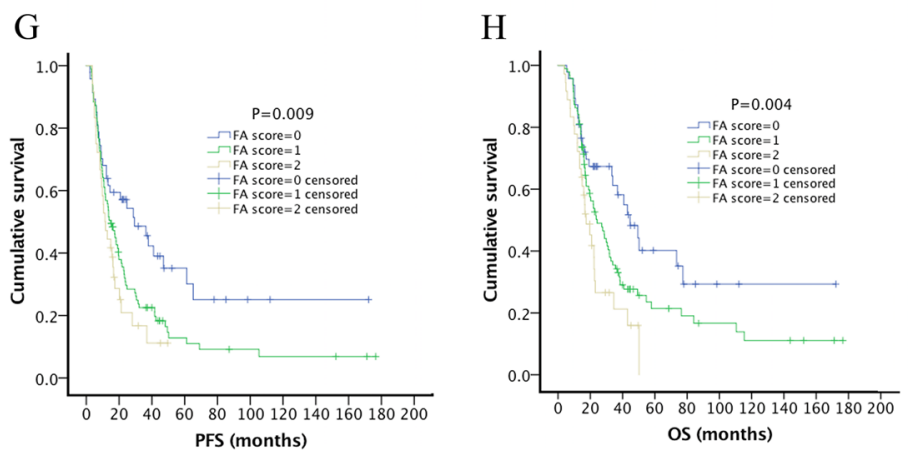

With aggressive treatment

Figure 2: Kaplan-Meier survival curves of different HGG subgroups. Kaplan-Meier method and log-rank test were used to investigate the prognostic value of pretreatment FA score in each subgroup. High FA score was significantly associated with better PFS and OS in subgroups of WHO grade III gliomas (A, PFS: $P<0.001 ; \mathbf{B}$, OS: $P<0.001)$ and WHO grade IV gliomas $(\mathbf{C}$, PFS: $P=0.002 ; \mathbf{D}$, OS: $P=0.001)$. Subgroup analysis based on treatment modalities showed that high pretreatment FA score still significantly predicted worse PFS and OS in patients received aggressive treatment $(\mathbf{E}$, PFS, $P=0.009 ; \mathbf{F}, \mathrm{OS}, P=0.004)$ or patients without aggressive treatment $(\mathbf{G}$, PFS, $P<0.001 ; \mathbf{H}$, OS, $P<0.001)$. 
Although the prognostic value of the pretreatment albumin level and fibrinogen level have been established in patients with cancers $[8,10-11,16-17]$, the mechanisms responsible for these associations are unknown. Fibrinogen detected using plasma is an important marker of systemic inflammatory. Fibrinogen can enhance tumor progression by inducing tumor cell proliferation, migration, and angiogenesis [19-21]. Fibrinogen is a common component of fibrinogen/fibrin matrix surrounding tumor cells, serving as a scaffold for binding members of growth factor families, such as transforming growth factor- $\beta$ (TGF- $\beta$ ), vascular endothelial growth factor (VEGF), fibroblast growth factor (FGF) and platelet-derived growth factor (PDGF) [22]. The binding of growth factors promotes tumor proliferation and stimulates angiogenesis [23].

Fibrinogen plays an important role in metastasis as well as facilitating stable adhesion, survival of metastatic emboli, or both, after tumor intravasation [24]. Fibrinogen deficiency can significantly diminish spontaneous hematogenous and lymphatic metastasis in the mice bearing levis lung carcinoma [24]. Adams et al reported that fibrinogen can support tumor growth as well as local invasion and metastasis, which contribute to the pathogenesis of colonic adenocarcinoma [25]. Moreover, fibrinogen may modulate the inflammatory response by inducing the production by peripheral blood mononuclear cells of the pro-inflammatory cytokines interleukin-1 (IL-1), interleukin-6 (IL-6), and tumor necrosis factor- $\alpha$ (TNF- $\alpha$ ) [26]. Moreover, inflammatory responses induced by fibrinogen in the tumor microenvironment are implicated in cancer progression [27].

In contrast, the level of circulating IL-6 was significantly higher in GBM patients compared with those of normal controls [28]. IL-6 is implicated in the regulation of VEGF secretion from glioblastoma cells, and VEGF can induce vascular permeability, which contributes to decreasing serum albumin levels [29]. Proinflammatory cytokines such as IL-1, IL-6 and TNF- $\alpha$ can down-regulate the hepatic synthesis of albumin [30]. The serum albumin level is inversely related to the host's systemic inflammatory response and nutritional status [31]. Therefore, a reduced albumin level may diminish the response of cancer patients to treatment and indicates poor survival. For these reasons, the FA score may serve as a prognostic indicator for patients with HGG.

Our study had certain limitations. First, this is a retrospective analysis with a single-center design, which may introduce selection bias. Further, the prognostic value of the FA score was not verified using a validation cohort. Second, data were unavailable for $I D H 1 / 2$ mutations, codeletion of chromosomes $1 \mathrm{p}$ and $19 \mathrm{q}$, TERT promoter mutations and ATRX mutation, which are prognostic factors for HGG [32]. Finally, because of its respective design, the PFS data might not be accurate. However, quantification of PFS is very difficult even in prospective trials because of pseudo-progression. Despite its limitations, we conclude that our study demonstrates the FA score serves as a significant prognostic indicator for managing patients with HGG. However, our findings must be validated by prospective trials.

In conclusion, we demonstrate for the first time that higher FA scores can significantly predict worse PFS and OS of patients with HGG. Fibrinogen and albumin levels are routinely measured in clinical practice. Thus, the FA score is an easily determined, noninvasive, economical indicator, which can facilitate predicting prognosis and guiding the administration of individualized treatment to patient with high-grade gliomas.

\section{MATERIALS AND METHODS}

\section{Ethical statement}

All patients have provided written informed consent for their information to be stored and used in the hospital database. Study approved was obtained from the Medical Ethics Committee of Sun Yat-sen University Cancer Center and the study was conducted in accordance with the ethical standard of the World Medical Association Declaration of Helsinki [33].

\section{Study population and data collection}

The inclusion criteria were as follows: (1) confirmation of WHO Grade III or IV glioma, (2) absence of other malignancies, (3) absence of other treatments before admission, and (4) integrity of clinical information and followed-up data. The exclusion criteria were as follows: (1) acute infection or chronic active inflammatory diseases, (2) glucocorticoid treatment before admission and (3) perioperative surgery-related mortality. Accordingly, we enrolled and retrospectively reviewed the records of 326 patients with histologically diagnosed high-grade gliomas who underwent tumor resection at Sun Yat-sen University Cancer Center from January 12001 to July 312014.

Baseline characteristics including demographics, KPS, pathological diagnosis, tumor grade, tumor size, tumor location, extent of resection and therapeutic information were collected using an electronic medical record system. All pathological specimens were reviewed and reclassified by central review according to WHO classification (revised in 2007) of central nervous system tumors [34]. High-grade gliomas were defined as WHO Grade III and IV gliomas. The tumor size was defined as the maximum diameter measured using pretreatment enhanced T1-weighted magnetic resonance imaging (MRI). For patients with multiple lesions, tumor size was calculated by adding the long diameter of all enhanced focus. Tumor location was categorized as cerebral cortex and non-cerebral cortex areas. 


\section{Treatment and follow up}

All patients underwent tumor resection at Sun Yatsen University Cancer Center and the extent of resection was classified as GTR, STR, partial resection and biopsy according to postoperative MRI and surgeon's notes. Aggressive adjuvant treatment was defined as fractionated radiotherapy plus adjuvant first-line chemotherapy or chemoradiotherapy plus adjuvant first-line chemotherapy post-operatively. First-line chemotherapy was categorized as temozolomide-based and nitrosourea/platinum-based strategies. A patient's refusal, financial problems, or poor condition excluded administration of aggressive treatment.

For all the patients, follow-up started from the date of surgery. Patients were generally followed quarterly for the first year, semiannually for the following 2 years and annually thereafter. During follow-up, patents were encouraged to undergo repeat contrast-enhanced MRI. Recording of medical history and physical examination were routinely performed. The last follow-up was until October 31, 2015 included verification of the clinical attendance records and direct telecommunications with the patients or their families. OS was measured from the date of surgery to the date of death from any cause, or the date of last follow-up visit. PFS was calculated from surgery to the first detection of progression, relapse, death from any cause, or the date of the last follow-up visit. Progression or relapse was identified according to the latest radiographic evidence.

\section{FA score}

Pretreatment plasma fibrinogen and serum albumin levels were determined using fasting serum sample after admission. The fibrinogen and albumin cut-off value were determined from receiver operating characteristic curves. Patients with elevated fibrinogen and decreased albumin levels were allocated to a score $=2$, those with one abnormal level were allocated to a score $=1$ and those with neither abnormal levels were allocated a score $=0$.

\section{Statistical analysis}

Differences of baseline and clinicopathological parameters between groups were evaluated by chi-square test or Fisher's exact test according to the distribution of data. The survival curves were calculated using the Kaplan-Meier method. Differences between the curves were evaluated using the log-rank test. The significant parameters identified by univariate analysis were evaluated using multivariate analysis employing the Cox proportional hazards model. All reported $p$ values were two-sided. $p<0.05$ was considered significant, and $95 \%$ CIs were calculated. All analyses were performed using the SPSS Statistics version 22.0 (IBM Corporation, Armonk, NY, United States).

\section{Authors' contributions}

ZQ He and H Duan participated in the design of the study, performed the statistical analysis and interpretation of data, and drafted the manuscript. F Al-Nahari, J Zhang and $\mathrm{ZH}$ Chen recruited patients to the study, collected and organized the data. CC Guo is an oncologist who contributed to perform chemotherapy for the patients. ZG Liu is a radiation oncologist who contributed to treat the patients. C Ke, XH Zhang, YS Chen, J Wang and ZP Chen are neurosurgeons who contributed to treat the patients. XB Jiang and YG Mou conceived of the study, revised and approved the final manuscript. All authors read and approved the final manuscript.

\section{ACKNOWLEDGMENTS}

This work was supported by Science and Technology Planning Project of Guangdong Province, China [grant number 2013B090500095, 2013B021800158, 2014A020212576, 2015B010125003]; National Natural Science Foundation of China [grant number 81201982, 81572500, 81672484]; Doctoral Fund of Ministry of Education of China [grant number 20120171120110]; Medical Scientific Research Foundation of Guangdong Province, China [grant number A2014247].

\section{CONFLICTS OF INTEREST}

The authors report no conflicts of interest in this work.

\section{REFERENCES}

1. Ostrom QT, Gittleman H, Fulop J, Liu M, Blanda R, Kromer C, Wolinsky Y, Kruchko C, Barnholtz-Sloan JS. CBTRUS Statistical Report: Primary Brain and Central Nervous System Tumors Diagnosed in the United States in 2008-2012. Neuro Oncol. 2015; 17: iv1-iv62. doi: 10.1093/ neuonc/nov189.

2. Wen PY, Kesari S. Malignant gliomas in adults. N Engl J Med. 2008; 359:492-507. doi: 10.1056/NEJMra0708126.

3. Gilbert MR, Dignam JJ, Armstrong TS, Wefel JS, Blumenthal DT, Vogelbaum MA, Colman H, Chakravarti A, Pugh S, Won M, Jeraj R, Brown PD, Jaeckle KA, et al. A Randomized Trial of Bevacizumab for Newly Diagnosed Glioblastoma. N Engl J Med. 2014; 370:699-708. doi: doi:10.1056/NEJMoa1308573.

4. He ZQ, Ke C, Al-Nahari F, Duan H, Guo CC, Wang Y, Zhang XH, Chen YS, Liu ZG, Wang J, Chen ZP, Jiang XB, Mou YG. Low preoperative prognostic nutritional index predicts poor survival in patients with newly diagnosed high-grade gliomas. J Neurooncol. 2017; 132:239-47. doi: 10.1007/s11060-016-2361-0. 
5. Bambury RM, Teo MY, Power DG, Yusuf A, Murray S, Battley JE, Drake C, O’Dea P, Bermingham N, Keohane C, Grossman SA, Moylan EJ, O'Reilly S. The association of pre-treatment neutrophil to lymphocyte ratio with overall survival in patients with glioblastoma multiforme. J Neurooncol. 2013; 114:149-54. doi: 10.1007/s11060-0131164-9.

6. Perisanidis C, Psyrri A, Cohen EE, Engelmann J, Heinze G, Perisanidis B, Stift A, Filipits M, Kornek G, Nkenke E. Prognostic role of pretreatment plasma fibrinogen in patients with solid tumors: A systematic review and meta-analysis. Cancer Treat Rev. 2015; 41:960-70. doi: 10.1016/j.ctrv.2015.10.002.

7. Lee JH, Hyun JH, Kim DY, Yoo BC, Park JW, Kim SY, Chang HJ, Kim BC, Kim TH, Oh JH, Sohn DK. The role of fibrinogen as a predictor in preoperative chemoradiation for rectal cancer. Ann Surg Oncol. 2015; 22:209-15. doi: 10.1245/s10434-014-3962-5.

8. Zhao J, Zhao M, Jin B, Yu P, Hu X, Teng Y, Zhang J, Luo Y, Zhang L, Zheng S, Zhou Q, Li H, Liu Y, et al. Tumor response and survival in patients with advanced non-smallcell lung cancer: the predictive value of chemotherapyinduced changes in fibrinogen. BMC Cancer. 2012; 12:330. doi: 10.1186/1471-2407-12-330.

9. Pichler M, Hutterer GC, Stojakovic T, Mannweiler S, Pummer K, Zigeuner R. High plasma fibrinogen level represents an independent negative prognostic factor regarding cancer-specific, metastasis-free, as well as overall survival in a European cohort of non-metastatic renal cell carcinoma patients. Br J Cancer. 2013; 109:1123-9. doi: 10.1038/bjc.2013.443.

10. Wen J, Yang Y, Ye F, Huang X, Li S, Wang Q, Xie X. The preoperative plasma fibrinogen level is an independent prognostic factor for overall survival of breast cancer patients who underwent surgical treatment. Breast. 2015; 24:745-50. doi: 10.1016/j.breast.2015.09.007.

11. Son HJ, Park JW, Chang HJ, Kim DY, Kim BC, Kim SY, Park SC, Choi HS, Oh JH. Preoperative plasma hyperfibrinogenemia is predictive of poor prognosis in patients with nonmetastatic colon cancer. Ann Surg Oncol. 2013; 20:2908-13. doi: 10.1245/s10434-013-2968-8.

12. Bairey O, Shacham-Abulafia A, Shpilberg O, Gurion R. Serum albumin level at diagnosis of diffuse large B-cell lymphoma: an important simple prognostic factor. Hematol Oncol. 2015; 34:184-192. doi: 10.1002/hon.2233.

13. Gupta D, Lis CG. Pretreatment serum albumin as a predictor of cancer survival: a systematic review of the epidemiological literature. Nutr J. 2010; 9:1. doi: 10.1186/1475-2891-9-69.

14. Borg N, Guilfoyle MR, Greenberg DC, Watts C, Thomson S. Serum albumin and survival in glioblastoma multiforme. J Neurooncol. 2011; 105:77-81. doi:10.1007/ s11060-011-0562-0.

15. Matsuda S, Takeuchi H, Kawakubo H, Fukuda K, Nakamura R, Takahashi T, Wada N, Saikawa Y, Omori T,
Kitagawa Y. Cumulative prognostic scores based on plasma fibrinogen and serum albumin levels in esophageal cancer patients treated with transthoracic esophagectomy: comparison with the Glasgow prognostic score. Ann Surg Oncol. 2015; 22:302-10. doi: 10.1245/s10434-014-3857-5.

16. Erdem S, Amasyali AS, Aytac O, Onem K, Issever H, Sanli O. Increased preoperative levels of plasma fibrinogen and $\mathrm{D}$ dimer in patients with renal cell carcinoma is associated with poor survival and adverse tumor characteristics. Urol Oncol. 2014; 32:1031-40. doi: 10.1016/j.urolonc.2014.03.013.

17. Polterauer S, Grimm C, Seebacher V, Concin N, Marth C, Tomovski C, Husslein H, Leipold H, Hefler-Frischmuth K, Tempfer C, Reinthaller A, Hefler L. Plasma fibrinogen levels and prognosis in patients with ovarian cancer: a multicenter study. Oncologist. 2009; 14:979-85. doi: 10.1634/theoncologist.2009-0079.

18. Schwartzbaum JA, Lal P, Evanoff W, Mamrak S, Yates A, Barnett GH, Goodman J, Fisher JL. Presurgical serum albumin levels predict survival time from glioblastoma multiforme. J Neurooncol. 1999; 43:35-41. doi:10.1023/A:1006269413998.

19. Staton CA, Brown NJ, Lewis CE. The role of fibrinogen and related fragments in tumour angiogenesis and metastasis. Expert Opin Biol Ther. 2003; 3:1105-20. doi: 10.1517/14712598.3.7.1105.

20. Steinbrecher KA, Horowitz NA, Blevins EA, Barney KA, Shaw MA, Harmel-Laws E, Finkelman FD, Flick MJ, Pinkerton MD, Talmage KE. Colitis-associated cancer is dependent on the interplay between the hemostatic and inflammatory systems and supported by integrin $\alpha \mathrm{M} \beta 2$ engagement of fibrinogen. Cancer Res. 2010; 70:2634-43. doi: 10.1158/0008-5472.CAN-09-3465.

21. Martino MM, Briquez PS, Ranga A, Lutolf MP, Hubbell JA. Heparin-binding domain of fibrin (ogen) binds growth factors and promotes tissue repair when incorporated within a synthetic matrix. Proc Natl Acad Sci U S A. 2013; 110:4563-8. doi: 10.1073/pnas.1221602110.

22. Simpson-Haidaris PJ, Rybarczyk B. Tumors and fibrinogen. Ann N Y Acad Sci. 2001; 936:406-25. doi: 10.1111/j.17496632.2001.tb03525.x.

23. Witsch E, Sela M, Yarden Y. Roles for growth factors in cancer progression. Physiology. 2010; 25:85-101. doi: 10.1152/physiol.00045.2009.

24. Palumbo JS, Potter JM, Kaplan LS, Talmage K, Jackson DG, Degen JL. Spontaneous Hematogenous and Lymphatic Metastasis, but not Primary Tumor Growth or Angiogenesis, Is Diminished in Fibrinogen-deficient Mice. Cancer Research. 2002; 62:6966.

25. Adams GN, Rosenfeldt L, Frederick M, Miller W, Waltz D, Kombrinck K, McElhinney KE, Flick MJ, Monia BP, Revenko AS, Palumbo JS. Colon Cancer Growth and Dissemination Relies upon Thrombin, Stromal PAR1, and Fibrinogen. Cancer Res. 2015; 75:4235-43. doi: 10.1158/0008-5472.CAN-15-0964. 
26. Jensen T, Kierulf P, Sandset PM, Klingenberg O, Joø GB, Godal HC, Skjønsberg OH. Fibrinogen and fibrin induce synthesis of proinflammatory cytokines from isolated peripheral blood mononuclear cells. Thromb Haemost. 2007; 97:822-9. doi: 10.1160/TH07-01-0039.

27. Jennewein C, Tran N, Paulus P, Ellinghaus P, Eble JA, Zacharowski K. Novel aspects of fibrin(ogen) fragments in the course of inflammation. Mol Med. 2011; 17:568-73. doi: 10.2119/molmed.2010.00146.

28. Reynés G, Vila V, Martín M, Parada A, Fleitas T, Reganon E, Martínez-Sales V. Circulating markers of angiogenesis, inflammation, and coagulation in patients with glioblastoma. J Neurooncol. 2011; 102:35-41. doi:10.1007/s11060-010-0290-X.

29. Loeffler S, Fayard B, Weis J, Weissenberger J. Interleukin-6 induces transcriptional activation of vascular endothelial growth factor (VEGF) in astrocytes in vivo and regulates VEGF promoter activity in glioblastoma cells via direct interaction between STAT3 and Sp1. Int J Oncol. 2005; 115:202-13. doi: 10.1002/ijc.20871.

30. Rothschild MA, Oratz M, Schreiber SS. Serum albumin. Hepatology. 1988; 8:385-401. doi: 10.1002/ hep. 1840080234.
31. Don BR, Kaysen G. Poor nutritional status and inflammation: serum albumin: relationship to inflammation and nutrition. Semin Dial. 2004; 17:432-7. doi: 10.1111/j.0894-0959.2004.17603.x.

32. Eckel-Passow JE, Lachance DH, Molinaro AM, Walsh KM, Decker PA, Sicotte H, Pekmezci M, Rice T, Kosel ML, Smirnov IV, Sarkar G, Caron AA, Kollmeyer TM, et al. Glioma Groups Based on 1p/19q, IDH, and TERT Promoter Mutations in Tumors. N Engl J Med. 2015; 372:2499-508. doi: 10.1056/NEJMoa1407279.

33. World Medical Association. World medical association declaration of helsinki: Ethical principles for medical research involving human subjects. JAMA. 2013; 310:2191-4. doi: 10.1001/jama.2013.281053.

34. Louis DN, Ohgaki H, Wiestler OD, Cavenee WK, Burger PC, Jouvet A, Scheithauer BW, Kleihues P. The 2007 WHO classification of tumours of the central nervous system. Acta Neuropathol. 2007; 114:97-109. doi: 10.1007/ s00401-007-0243-4. 